\title{
Sinus Node Dysfunction
}

National Cancer Institute

\section{Source}

National Cancer Institute. Sinus Node Dysfunction. NCI Thesaurus. Code C62243.

A derangement in the normal functioning of the sinoatrial node. Typically, SA node dysfunction is manifest as sinoatrial exit block or sinus arrest, but may present as an absolute or relative bradycardia in the presence of a stressor. It may be associated with bradycardia-tachycardia syndrome 